\title{
Design, Synthesis and Antibacterial Studies of Some New Pyridopyrimidine Derivatives as Biotin Carboxylase Inhibitors
}

\author{
Original \\ Article \\ Vivek B. Panchabhai, Parag G. Ingole, S. R. Butle \\ School of Pharmacy, Swami Ramanand Teerth Marathwada University, Nanded, \\ Maharashtra, India.
}

\begin{abstract}
Present study reports the development of novel pyridopyrimidine derivatives as biotin carboxylase inhibitors with potent antibacterial activity. These compounds were designed to avoid possibility of resistance development. Accordingly eighteen compounds were synthesised and characterized on the basis of spectral data. These compounds were tested for their antibacterial potential by the enzyme kinetic assay against the biotin carboxylase. The minimum inhibitory concentration (MIC) and single step resistance studies were also performed. Compound 2-((2-Phenylpyrido[2,3-d] pyrimidin-4-yl)amino)phenol (6o) showed promising activity in biotin carboxylase inhibition with low MIC. It showed molecular docking score of -7.96, this compound showed formation of hydrogen bonds with the active site residues and van Der Walls interactions. The MIC of compounds under investigation was in the rage of $2-5 \mu \mathrm{g} / \mathrm{mL}$ over most of the strains studied. It also showed the mutant selection windows of around five which is better than the reference compound rifampin. This compound 60 can be studied further and developed into a potential antibacterial lead molecule.
\end{abstract}

Key Words: Biotin carboxylase, drug resistance, molecular docking, pyridopyrimidine.

Received: 02 March 2019, Accepted: 30 June 2019

Corresponding Author: Vivek B. Panchabhai, School of Pharmacy, Swami Ramanand Teerth Marathwada University,

Nanded, Maharashtra, India, Tel.:: +919423714528, E-mail: vivekpanchabhai82@gmail.com

ISSN: 1110-0930, xxx 2020 Vol.58, No. 1

\section{INTRODUCTION}

In 2014, the World Health Organization (WHO) released an alarming report on microbial resistance to antibiotics especially in bacteria. The WHO report show a comprehensive picture of data obtained from 114 countries. ${ }^{[1]}$ According to it at least seven different common bacteria cause serious diseases like postoperative infections, blood stream infections, hospital acquired infections and gonorrhoea have become drug resistant. Lot of information regarding the spread of drug resistant microorganisms are available still a big gap is observed in tracking antibiotic resistance. United States alone reported over two million deaths due to bacterial infection and 23,000 deaths due to drug resistant bacterial strains. ${ }^{[2]}$ One of the major reasons for rise of drug resistance is lack of new group of antibiotics. The penicillin was discovered during late 1920 s, followed by cephalosporin in late 1940s. After a gap of two decades carbapenams and fluoroquinolones were discovered in the 80s. However, since then no such breakthrough discovery has been reported. ${ }^{[3]}$ It is known that about 30-40 targets are generally harnessed for drug discovery process against the bacteria, thus there is an urgent need for smart molecules that target novel targets or multiple targets. ${ }^{[4]}$
The development of techniques like virtual screening, pharmacophore generation, and fragment based drug discovery, advances in drug delivery has resulted in discovery of newer compounds and validation of newer targets and their proper administration to patients. ${ }^{[5,6]}$ One of the major metabolic pathways found in bacteria is the fatty acid synthesis pathway and has become a popular target among medicinal chemist. In a recent development, the enzyme biotin carboxylase (BC) was found to be a very attractive target for inhibiting the Acetyl CoA carboxylase mediated reaction. $\mathrm{BC}$ plays a vital role in Acetyl CoA carboxylase (ACC) catalysed reactions. Biotin carboxylases catalyse the carboxylation of biotin carboxyl carrier protein (BCCP)-biotin in the presence of bicarbonate to form the BCCP-biotin- $\mathrm{CO}_{2}$. In the next step, BCCPbiotin- $\mathrm{CO}_{2}$ transfer the carboxyl group to Acetyl-CoA and forms malonyl-CoA in presence of carboxyltransferase (CT) (Figure 1). ${ }^{[7]}$ The heterocyclic moieties like the pyridopyrimidines such as $i$ to iv were reported to possess significant BC inhibitory activity (Figure 2).$^{[8]}$ Mochalkin et al. showed that this class of compounds bind to the ATP binding site of bacterial $\mathrm{BC}$ did not bind or inhibit the human BC. ${ }^{[9]}$ Several recent reports on the development of $\mathrm{BC}$ inhibitors have validated it as a potential drug target for the development of novel antibacterial compounds. 
Earlier, we have reported the development of various heterocyclic scaffolds like pyrimidines and benzazoles as anti-bacterial and anti-tubercular agents. ${ }^{[10-12]}$ In continuation to our efforts we aim to hit this newly validated target for the development of novel antibacterial agents on the basis of structural analogy and molecular docking studies. The pyridopyrimidine scaffolds provides for a wide range of activity like Bcr-abl inhibitors ${ }^{[13]}$, MexAB-OprM efflux pump inhibitor ${ }^{[14]}$, Adenosine kinase inhibitors ${ }^{[15]}$, $\mathrm{PI} 3 \mathrm{~K} / \mathrm{mTOR}$ dual inihibition ${ }^{[16]}$, Jak1/2 inhibitor ${ }^{[17]}$, cell cycle inhibition ${ }^{[18]}$, antitubercular activity ${ }^{[19,20]}$, antioxidant activity $^{[21]}$ and antibacterial activity. ${ }^{[9,22-27]}$ Herein, we report the synthesis, antibacterial evaluation with resistance studies of some novel pyridopyrimidine derivatives and molecular docking analysis of the synthesised compounds.

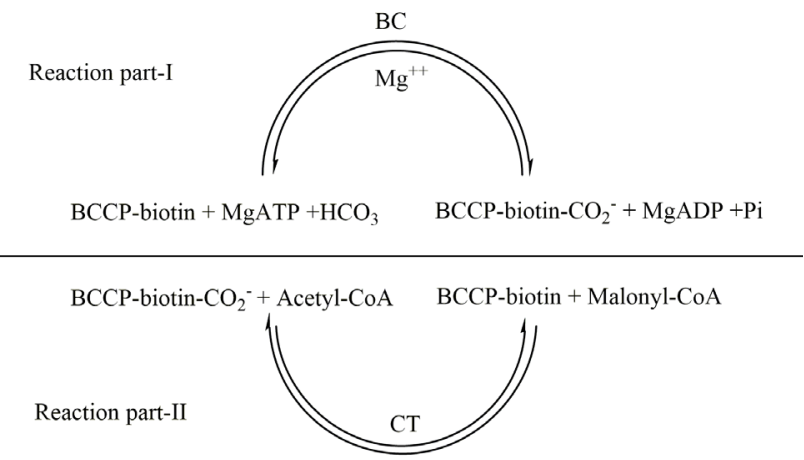

Fig. 1: Reactions catalysed by biotin carboxylase and carboxyltransferase<smiles>Nc1ncc2cc(-c3c(Br)cccc3Br)c(N)nc2n1</smiles><smiles>COc1cccc(OC)c1-c1cc2cnc(N)nc2nc1N</smiles><smiles>Nc1ncc2cc(-c3ccccc3)c(N3CCCC3)nc2n1</smiles>

iii<smiles>CCN(CC)CCCCNc1ncc2cc(-c3cc(OC)cc(OC)c3)c(NC(=O)NC(C)(C)C)nc2n1</smiles>

Fig. 2: Pyridopyrimidine inhibitors of biotin carboxylase

\section{Experimental}

Chemicals, assay kits, reagents and materials were obtained from Sigma Aldrich, Germany, USA and Alfa Aeser, UK. Analytical grade solvents were obtained from the E. Merck, India. Melting points (mp) were detected with open capillaries using Thermonik Precision Melting point cum Boiling point apparatus (C-PMB-2, Mumbai, India) and are uncorrected. Chromatography of the synthesized intermediates and title compounds were performed on silica gel pre-coated plates (Merck: 100-200 mesh). Thin layer chromatography (TLC) was used to monitor the progress and/or completion of the reactions. IR spectra (KBr) were recorded on FTIR-8400s spectrophotometer (Shimadzu, Japan). ${ }^{1} \mathrm{H}$ and ${ }^{13} \mathrm{C}$ NMR was obtained using a Bruker Advance-II 400 Spectrometer on $400 \mathrm{MHz}$ using tetramethylsilane (TMS) as internal standard. All chemical shift values were recorded as $\delta(\mathrm{ppm})$, coupling constant value $\mathrm{J}$ is measured in hertz, the peaks are presented as s (singlet), d (doublet), t (triplet), br s (broad singlet), dd (double doublet), m (multiplet). The purity of compounds was controlled by thin layer chromatography (Merck, silica gel, HF254-361, type 60, 0.25 mm, Darmstadt, Germany). Mass spectra (ESI-MS) were recorded at ESI-MS spectrometer (DPX-400, Bruker, USA). Shimadzu High Performance Liquid Chromatography (HPLC) system with SPD-20A prominence Photodiode Array (PDA) detector and LC-20AB prominence LC solution software were used in this study.

\subsection{Materials and Methods}

\subsubsection{Synthesis of methyl 2-aminonicotinate(2)}

2-Aminopyridine 3-carboxyllic acid ${ }^{[1]}$ (13.8g, 0.1 mole) was dissolved in $400 \mathrm{~mL}$ of anhydrous methanol, to this solution thionyl chloride $(11.8 \mathrm{~mL}, 14.03 \mathrm{~g}, 0.1 \mathrm{~mole})$ was added dropwise for half hour with constant stirring. On complete addition, it was refluxed for 12 hours after which the reaction mixture was cooled to room temperature. The solvent from the mixture was evaporated under vacuum and remaining residue was dissolved in chloroform. The chloroform layer was collected and washed with $5 \% \mathrm{HCl}$ and $10 \% \mathrm{NaHCO}_{3}$ followed by water. This layer was further dried over sodium sulphate and concentrated to give methyl 2-aminonicotinate.

Yield: $71 \%$. mp: $126-129^{\circ} \mathrm{C}$ (ethanol). IR (KBr) $\mathrm{cm}^{-1}: 3424.1,3319.2,1655.1,1618.5,1601.1 .{ }^{1} \mathrm{H}$ NMR (DMSO-d6, $400 \mathrm{MHz}) \delta: 3.86(\mathrm{~s}, 3 \mathrm{H}, \mathrm{CH} 3), 7.0(\mathrm{~d}, 1 \mathrm{H}$, $\mathrm{J}=7.01$, Ar), 7.44(dd, 1H, J=7.45, Ar), 8.69(s, 1H, Ar), 12.19(bs, 2H, NH2). ${ }^{13} \mathrm{C}$ NMR (DMSO-d6, $100 \mathrm{MHz}$ ) $\delta: 51.5,110.0,111.6,139.7,155.4,158.0,167.5$. HRMS (EI) $\mathrm{m} / \mathrm{z}$ calcd for $\mathrm{C}_{7} \mathrm{H}_{8} \mathrm{~N}_{2} \mathrm{O}_{2}$ 152.1530, found 152.1525 . Anal: C (55.26/55.11), H (5.30/5.25), N (18.41/18.37), $\mathrm{O}(21.03 / 21.09)$.

\subsubsection{Synthesis of methyl 2-benzamidonicotinate(3)}

To a solution of methyl 2-aminonicotinate $(7.60 \mathrm{~g}, 0.5$ mole) in $50 \mathrm{~mL}$ chloroform was added triethylamine $(2.5$ $\mathrm{mL}, 0.2 \mathrm{~mole}$ ). To this solution 3-chlorobenzoyl chloride (3.04 mL, 2.22g, 0.02 mole) in chloroform was added dropwise over a period of one hour with continues stirring. It was further stirred on room temperature for next 20 hours. After that the mixture was filtered to remove any residue formed, the solution was washed with $10 \% \mathrm{NaHCO}_{3}$ and $5 \% \mathrm{HCl}$. It was further treated with ethyl acetate in presence of hexane to give methyl-2-benzamidonicotinate as solid product. 
Yield: $64 \%$. mp: $122-124^{\circ} \mathrm{C}$ (ethanol). IR $(\mathrm{KBr}) \mathrm{cm}^{-1}$ : 3320.6, 1900.7, 1845.6, 1742.2, 1715.3, 1651.2, 1455.1. ${ }^{1} \mathrm{H}$ NMR (DMSO-d6, $400 \mathrm{MHz}$ ) $\delta: 3.77$ (s, 3H, CH3), 7.7 (d, $2 \mathrm{H}, \mathrm{J}=7.78, \operatorname{Ar}$ ), 7.8 (s, 1H, Ar), 8.13 (d, 2H, J=7.12, Ar), 8.3 (d, 1H, J=7.26, Ar), 8.3 (dd, 2H, J=7.38, Ar), 12.73 (bs, $1 \mathrm{H}, \mathrm{NH}) .{ }^{13} \mathrm{C}$ NMR (DMSO-d6, $\left.100 \mathrm{MHz}\right) \delta: 51.5,110.4$, 111.2 , 127.1, 132.6, 134.7, 137.4, 148.2, 155.5, 167.2, 168.1. HRMS (EI) $\mathrm{m} / \mathrm{z}$ calcd for $\mathrm{C}_{14} \mathrm{H}_{12} \mathrm{~N}_{2} \mathrm{O}_{3}$ 256.0431, found 256.0459. Anal: C (65.62/65.68), H (4.72/4.60), N (10.62/10.67), O (18.73/18.39).

\subsubsection{Synthesis of 2-phenylpyrido[2,3-d]pyrimidin- 4(3H)-one(4)}

Compound 3 (5.12g, 0.02 mole) was dissolved in 10 $\mathrm{mL}$ of methanol and to this a $28 \%$ solution of $\mathrm{NH}_{3} \mathrm{OH}$ was added. This mixture was then heated for 5 hours and then $40 \mathrm{~mL}$ of $\mathrm{NaOH}$ was added with vigorous stirring followed by refluxing for 12 hours. It was allowed to cool down to room temperature leading to formation of a precipitate which was further filtered and dried to obtain white solid as 2-phenylpyrido[2,3-d]pyrimidin-4(3H)-one.

Yield: $78 \%$. mp: $168-170^{\circ} \mathrm{C}$ (ethanol). IR $(\mathrm{KBr}) \mathrm{cm}^{-1}$ : 3420.1, 3318.2, 1715.3, 1657.4, 1655.1, 1618.5. ${ }^{1} \mathrm{H}$ NMR (DMSO-d6, $400 \mathrm{MHz}) \delta: 5.8$ (bs, 1H, Ar-NH), 6.8 (m, 1H, Ar), 6.8 (m, 5H, Ar), 7.15 (t, 2H, J=7.60, Ar). ${ }^{13} \mathrm{C}$ NMR (DMSO-d6, $100 \mathrm{MHz}) \delta: 115.4,120.09,128.2,129.10$, 130.18, 132.40, 136.7, 156.0, 157.5, 159.2, 161.0. HRMS (EI) $\mathrm{m} / \mathrm{z}$ calcd for $\mathrm{C}_{13} \mathrm{H}_{9} \mathrm{~N}_{3} \mathrm{O} 233.0751$, found 233.0772. Anal: C (69.95/69.80), H (4.06/4.03), N (18.82/18.67), $\mathrm{O}(7.17 / 7.14)$.

\subsubsection{Synthesis of 4-chloro-2-phenylpyrido[2,3-d] pyrimidine(5)}

Compound 4 (2.23 g, 0.01 mole) was refluxed with phosphorus oxychloride for five hour during which all the starting material was dissolved and utilised. Excess of phosphorus oxychloride was removed under vacuum and the remaining residue was poured in ice-cold water and chloroform. The chloroform layer was separated and treated with $\mathrm{NaHCO}_{2}$. The chloroform layer was further washed with brine and then purified using silica gel column with chloroform as an eluent to provide 4-chloro2-phenylpyrido[2,3-d]pyrimidine.

Yield: $67 \%$. mp: $156-158^{\circ} \mathrm{C}$ (ethanol). IR $(\mathrm{KBr}) \mathrm{cm}^{-1}$ : 3423.1, 3376.2, 3319.2, 1657.4, 1655.1, 1619.8. ${ }^{1} \mathrm{H}$ NMR (DMSO-d6, $400 \mathrm{MHz}) \delta: 6.9$ (s, 1H, Ar), 7.4 (m, 1H, Ar), 7.49 (m, 2H, Ar), 7.7 (m, 1H, Ar), 7.76 (m, 1H, Ar), 8.01 (t, 2H, J=8.50, Ar). ${ }^{13} \mathrm{C}$ NMR (DMSO-d6, $\left.100 \mathrm{MHz}\right) \delta$ : $118.4,121.09,128.8,129.40,130.13,132.40,134.7,156.0$, 157.5, 159.2, 160.10. HRMS (EI) $\mathrm{m} / \mathrm{z}$ calcd for $\mathrm{C}_{13} \mathrm{H}_{8} \mathrm{ClN}_{3}$ 241.0407, found 241.0410. Anal: C (64.61/64.50), H (3.34/3.39), Cl (14.65/14.67), N (17.39/17.30).

\subsubsection{General Synthesis of 2-phenyl-N- (substituted phenyl) pyrido[2,3-d] pyrimidin-4- amine (6a-r)}

Compound 5 was reacted with various anilines in $4 \mathrm{~mL}$ of dry DMF, to this solution 86 microliters of diisopropylamine (DIPA) was added and heated over a period of 3-6 hours. This reaction mixture was cooled to RT and residue was filtered out and purified using column chromatography to yield respective derivatives. Total eighteen derivatives were synthesised, these compounds are enlisted as follows;

\subsubsection{N-(4-Fluorophenyl)-2-phenylpyrido[2,3-d]} pyrimidin-4-amine (6a)

Yield: $59 \%$. mp: $152-154^{\circ} \mathrm{C}$ (ethanol). IR (KBr) $\mathrm{cm}^{-1}$ : 3422.1, 3373.2, 3315.2, 1652.4, 1654.1, 1618.8. ${ }^{1} \mathrm{H}$ NMR (DMSO-d6, $400 \mathrm{MHz}) \delta: 6.8(\mathrm{~m}, 1 \mathrm{H}, \mathrm{NH}), 7.3(\mathrm{~m}, 2 \mathrm{H}, \mathrm{Ar})$, 7.36(m, 2H, Ar), 7.41(m, 1H, Ar), 7.59(m, 2H, Ar), 8.33(t, $3 \mathrm{H}, \mathrm{J}=7.76, \mathrm{Ar}), 8.59$ (dd, $1 \mathrm{H}, \mathrm{J}=7.26, \mathrm{Ar}) .{ }^{13} \mathrm{C} \mathrm{NMR}$ (DMSO-d6, $100 \mathrm{MHz}$ ) $\delta:$ 103.6, 114.0, 116.1, 120.7, 126.5, $127.1,129.2,130.1,131.5,136.7,153.0,155.1,157.9$, 159.0. HRMS (EI) $\mathrm{m} / \mathrm{z}$ calcd for C19H13FN4 316.1124, found 316.1126. Anal: $\mathrm{C}(72.14 / 72.17), \mathrm{H}(4.14 / 4.12)$, $\mathrm{F}(6.01 / 6.03), \mathrm{N}(17.71 / 17.67)$.

\subsubsection{N-(2-Fluorophenyl)-2-phenylpyrido[2,3-d] pyrimidin-4-amine (6b)}

Yield: $48 \%$. mp: $150-152^{\circ} \mathrm{C}$ (ethanol). IR $(\mathrm{KBr}) \mathrm{cm}^{-1}$ : 3421.1, 3376.4, 3319.5, 1657.6, 1655.3, 1619.1. ${ }^{1} \mathrm{H}$ NMR (DMSO-d6, $400 \mathrm{MHz}$ ) $\delta:$ 7.12(m, 2H, Ar), 7.27(m, 2H, Ar), 7.5(m, 2H, Ar), 7.8(m, 2H, Ar), 8.11(s, 2H, Ar), 8.13(d, $2 \mathrm{H}, \mathrm{Ar}), 12.63(\mathrm{bs}, 1 \mathrm{H}, \mathrm{NH}) .{ }^{13} \mathrm{C}$ NMR (DMSO-d6, 100 MHz) $\delta: 103.6,114.0,116.1,120.7,126.5,127.1,129.2$, $130.1,131.5,136.7,153.0,155.1,157.9,159.0$. HRMS (EI) $\mathrm{m} / \mathrm{z}$ calcd for $\mathrm{C}_{10} \mathrm{H}_{13} \mathrm{FN}_{4} 316.1124$, found 316.1126 . Anal: C (72.14/72.17), H (4.14/4.12), F (6.01/6.03), N (17.71/17.67).

\subsubsection{N-(4-Chlorophenyl)-2-phenylpyrido[2,3-d] pyrimidin-4-amine (6c)}

Yield: $64 \%$. mp: $149-151^{\circ} \mathrm{C}$ (ethanol). IR (KBr) $\mathrm{cm}^{-1}: 3420.4,3375.4,3317.5,1656.7,1656.3,1618.8$. ${ }^{1} \mathrm{H}$ NMR (DMSO-d6, $\left.400 \mathrm{MHz}\right) \delta: 6.8(\mathrm{~m}, 1 \mathrm{H}, \mathrm{NH})$, 7.31(m, 2H, Ar), 7.36(m, 2H, Ar), 7.41(m, 1H, Ar), 7.59(m, $2 \mathrm{H}, \mathrm{Ar}), 8.33(\mathrm{t}, 3 \mathrm{H}, \mathrm{J}=7.76, \mathrm{Ar}), 8.59(\mathrm{dd}, 1 \mathrm{H}, \mathrm{J}=7.26, \mathrm{Ar})$. ${ }^{13} \mathrm{C}$ NMR (DMSO-d6, $\left.100 \mathrm{MHz}\right) \delta: 103.6,114.0,116.1$, $120.7,126.5,127.1,129.2,130.1,131.5,136.7,153.0$, 155.1, 157.9, 159.0. HRMS (EI) $\mathrm{m} / \mathrm{z}$ calcd for $\mathrm{C}_{19} \mathrm{H}_{13} \mathrm{ClN}_{4}$ 332.0829, found 332.0835. Anal: $\mathrm{C}(68.50 / 68.57)$, $\mathrm{H}(3.94 / 3.90), \mathrm{Cl}(10.65), \mathrm{N}(16.84 / 16.88)$. 


\subsubsection{N-(2-Chlorophenyl)-2-phenylpyrido[2,3-d]} pyrimidin-4-amine (6d)

Yield: $60 \%$. mp: $147-149^{\circ} \mathrm{C}$ (ethanol). IR (KBr) $\mathrm{cm}^{-1}$ : 3423.6, 3376.3, 3319.5, 1657.3, 1655.2, 1619.7. ${ }^{1} \mathrm{H}$ NMR (DMSO-d6, $400 \mathrm{MHz}) \delta: 6.8(\mathrm{~m}, 1 \mathrm{H}, \mathrm{NH}), 7.31(\mathrm{~m}$, 2H, Ar), $7.36(\mathrm{~m}, 2 \mathrm{H}, \mathrm{Ar}), 7.4(\mathrm{~m}, 1 \mathrm{H}, \mathrm{Ar}), 7.59(\mathrm{~m}, 2 \mathrm{H}$, Ar), 8.33(t, 3H, J=7.76, Ar), 8.59(dd, 1H, J=7.26, Ar). ${ }^{13} \mathrm{C}$ NMR (DMSO-d6, $\left.100 \mathrm{MHz}\right) \delta: 103.6,114.0,116.1$, $120.7,126.5,127.1,129.2,130.1,131.5,136.7,153.0$, 155.1, 157.9, 159.0. HRMS (EI) $\mathrm{m} / \mathrm{z}$ calcd for $\mathrm{C}_{19} \mathrm{H}_{13} \mathrm{ClN}_{4}$ 332.0829, found 332.0835. Anal: C (68.55/68.57), H (3.90/3.94), Cl (10.65), N (16.84/16.80).

\subsubsection{N-(4-Bromophenyl)-2-phenylpyrido[2,3-d]} pyrimidin-4-amine (6e)

Yield: $51 \%$. mp: $156-158^{\circ} \mathrm{C}$ (ethanol). IR $(\mathrm{KBr}) \mathrm{cm}^{-1}$ : 3422.4, 3375.4, 3317.1, 1654.5, 1656.2, 1615.4. ${ }^{1} \mathrm{H}$ NMR (DMSO-d6, $400 \mathrm{MHz}) \delta(\mathrm{m}, 1 \mathrm{H}, \mathrm{NH}), 7.31(\mathrm{~m}, 2 \mathrm{H}, \mathrm{Ar})$, 7.36 (m, 2H, Ar), 7.41(m, 1H, Ar), 7.59(m, 2H, Ar), 8.33(t, $3 \mathrm{H}, \mathrm{J}=7.76, \mathrm{Ar}), 8.59$ (dd, $1 \mathrm{H}, \mathrm{J}=7.26, \mathrm{Ar}) .{ }^{13} \mathrm{C}$ NMR (DMSO-d6, $100 \mathrm{MHz}) \delta$ : 103.6, 114.0, 116.1, 120.7, 126.5, $127.1,129.2,130.1,131.5,136.7,153.0,155.1,157.9$, 159.0. HRMS (EI) $\mathrm{m} / \mathrm{z}$ calcd for $\mathrm{C}_{19} \mathrm{H}_{13} \mathrm{BrN}_{4} 376.0324$, found 376.0320. Anal: C (60.49/60.50), H (3.47/3.50), Br (21.18/21.14), N (14.85/14.87).

\subsubsection{N-(2-Bromophenyl)-2-phenylpyrido[2,3-d] pyrimidin-4-amine (6f)}

Yield: $50 \%$. mp: $159-161{ }^{\circ} \mathrm{C}$ (ethanol). IR $(\mathrm{KBr}) \mathrm{cm}^{-1}$ : 3422.4, 3375.4, 3317.1, 1654.5, 1656.2, 1615.4. ${ }^{1} \mathrm{H}$ NMR (DMSO-d6, $400 \mathrm{MHz}) \delta: 6.8(\mathrm{~m}, 1 \mathrm{H}, \mathrm{NH}), 7.31(\mathrm{~m}, 2 \mathrm{H}$, Ar), 7.36(m, 2H, Ar), 7.41(m, 1H, Ar), 7.59(m, 2H, Ar), 8.33(t, 3H, J=7.76, Ar), 8.59(dd, 1H, J=7.26, Ar). ${ }^{13} \mathrm{C}$ NMR (DMSO-d6, 100 MHz) $\delta:$ 103.6, 114.0, 116.1, 120.7, 126.5, $127.1,129.2,130.1,131.5,136.7,153.0,155.1,157.9$, 159.0. HRMS (EI) $\mathrm{m} / \mathrm{z}$ calcd for $\mathrm{C}_{19} \mathrm{H}_{13} \mathrm{BrN}_{4}$ 376.0324, found 376.0321. Anal: C (60.47/60.49), $\mathrm{H}(3.48 / 3.51), \mathrm{Br}$ (21.11/21.15), N (14.82/14.86).

\subsubsection{2-Phenyl-N-(4-(trifluoromethyl)phenyl) pyrido[2,3-d]pyrimidin-4-amine (6g)}

Yield: $65 \%$. mp: $158-160^{\circ} \mathrm{C}$ (ethanol). IR (KBr) $\mathrm{cm}^{-1}: 3423.1,3378.2,3317.2,1659.4,1654.1,1620.8 .{ }^{1} \mathrm{H}$ NMR (DMSO-d6, $400 \mathrm{MHz}) \delta: 6.8(\mathrm{~m}, 1 \mathrm{H}, \mathrm{NH}), 7.31(\mathrm{~m}$, $2 \mathrm{H}, \mathrm{Ar}), 7.36(\mathrm{~m}, 2 \mathrm{H}, \mathrm{Ar}), 7.41(\mathrm{~m}, 1 \mathrm{H}, \mathrm{Ar}), 7.59(\mathrm{~m}, 2 \mathrm{H}$, Ar), 8.33(t, 3H, J=7.76, Ar), 8.59(dd, $1 \mathrm{H}, \mathrm{J}=7.26$, Ar). ${ }^{13} \mathrm{C}$ NMR (DMSO-d6, 100 MHz) $\delta: 103.6,114.0,116.1,120.7$, $124.1,126.5,127.1,129.2,130.1,131.5,136.7,153.0$, 155.1, 157.9, 159.0. HRMS (EI) $\mathrm{m} / \mathrm{z}$ calcd for $\mathrm{C}_{20} \mathrm{H}_{13} \mathrm{~F}_{3} \mathrm{~N}_{4}$ 366.3472, found 366.3211. Anal: $\mathrm{C}(65.57 / 65.60), \mathrm{H}$ (3.58/3.61), F (15.56/15.67), N (15.29/15.40).

\subsubsection{2-Phenyl-N-(p-tolyl)pyrido[2,3-d]pyrimi- din-4-amine (6h)}

Yield: $55 \%$. mp: $165-167^{\circ} \mathrm{C}$ (ethanol). IR (KBr) $\mathrm{cm}^{-1}$ : 3423.3, 3377.4, 3318.5, 1656.9, 1656.2, 1617.4. ${ }^{1} \mathrm{H}$ NMR (DMSO-d6, $400 \mathrm{MHz}) \delta: 2.18(\mathrm{~s}, 3 \mathrm{H}, \mathrm{CH} 3), 6.8(\mathrm{~m}, 1 \mathrm{H}$, $\mathrm{NH}), 7.31$ (m, 2H, Ar), 7.36(m, 2H, Ar), 7.41(m, 1H, Ar), 7.59(m, 2H, Ar), 8.33(t, 3H, J=7.76, Ar), 8.59(dd, $1 \mathrm{H}$, $\mathrm{J}=7.26, \mathrm{Ar}) .{ }^{13} \mathrm{C}$ NMR (DMSO-d6, $\left.100 \mathrm{MHz}\right) \delta$ : 103.6, $114.0,116.1,120.7,124.1,126.5,127.1,129.2,130.1$, 131.5, 136.7, 153.0, 155.1, 157.9, 159.0. HRMS (EI) m/z calcd for $\mathrm{C}_{20} \mathrm{H}_{16} \mathrm{~N}_{4} 312.1375$, found 312.1390. Anal: C (76.90/76.85), H (5.16/5.19), N (17.94/17.90).

\subsubsection{2-Phenyl-N-(m-tolyl)pyrido[2,3-d]pyrimi- din-4-amine (6i)}

Yield: $50 \%$. mp: $164-166^{\circ} \mathrm{C}$ (ethanol). IR $(\mathrm{KBr}) \mathrm{cm}^{-1}$ : 3423.1, 3376.2, 3319.2, 1657.4, 1655.1, 1619.8. ${ }^{1} \mathrm{H}$ NMR (DMSO-d6, $400 \mathrm{MHz}) \delta$ : 2.4(s, 3H, CH3), 6.8(m, 1H, NH), $7.11(\mathrm{t}, 1 \mathrm{H}, \mathrm{J}=7.82, \mathrm{Ar}), 7.31(\mathrm{~m}, 2 \mathrm{H}, \mathrm{Ar}), 7.36(\mathrm{~m}, 2 \mathrm{H}, \mathrm{Ar})$, 7.41(m, 1H, Ar), 7.59(m, 2H, Ar), 8.33(t, 3H, J=7.76, Ar), 8.59(dd, $1 \mathrm{H}, \mathrm{J}=7.26$, Ar). ${ }^{13} \mathrm{C}$ NMR (DMSO-d6, $100 \mathrm{MHz}$ ) $\delta: 103.6,114.0,116.1,120.7,124.1,126.5,127.1,129.2$, $130.1,131.5,136.7,153.0,155.1,157.9,159.0$. HRMS (EI) $\mathrm{m} / \mathrm{z}$ calcd for $\mathrm{C}_{20} \mathrm{H}_{16} \mathrm{~N}_{4}$ 312.1375, found 312.1399. Anal: C (76.90/76.84), H (5.16/5.18), N (17.94/17.91).

\subsubsection{2-Phenyl-N-(o-tolyl)pyrido[2,3-d]pyrimi- din-4-amine (6j)}

Yield: $58 \%$. mp: $160-162^{\circ} \mathrm{C}$ (ethanol). IR $(\mathrm{KBr}) \mathrm{cm}^{-1}$ : 3423.4, 3377.8, 3318.6, 1656.5, 1656.3, 1618.7. ${ }^{1} \mathrm{H}$ NMR (DMSO-d6, $400 \mathrm{MHz}) \delta: 2.12(\mathrm{~s}, 3 \mathrm{H}, \mathrm{CH} 3), 6.8(\mathrm{~m}, 1 \mathrm{H}$, $\mathrm{NH}), 7.11(\mathrm{t}, 1 \mathrm{H}, \mathrm{J}=7.82, \mathrm{Ar}), 7.31(\mathrm{~m}, 2 \mathrm{H}, \mathrm{Ar}), 7.36(\mathrm{~m}$, 2H, Ar), 7.41(m, 1H, Ar), 7.59(m, 2H, Ar), 8.33(t, 3H, $\mathrm{J}=7.76$, Ar), 8.59(dd, 1H, J=7.26, Ar). ${ }^{13} \mathrm{C}$ NMR (DMSOd6, $100 \mathrm{MHz}) \delta: 103.6,114.0,116.1,120.7,124.1,126.5$, $127.1,129.2,130.1,131.5,136.7,153.0,155.1,157.9$, 159.0. HRMS (EI) $\mathrm{m} / \mathrm{z}$ calcd for $\mathrm{C}_{20} \mathrm{H}_{16} \mathrm{~N}_{4} 312.1375$, found 312.1299. Anal: C (76.90/76.89), H (5.16/5.22), N (17.94/17.87)

\subsubsection{N-(4-Methoxyphenyl)-2-phenylpyrido[2,3- dlpyrimidin-4-amine (6k)}

Yield: $66 \%$. mp: $167-169^{\circ} \mathrm{C}$ (ethanol). IR $(\mathrm{KBr}) \mathrm{cm}^{-1}$ : 3425.3, 3378.2, 3319.7, 1656.5, 1656.3, 1618.8. ${ }^{1} \mathrm{H}$ NMR (DMSO-d6, $400 \mathrm{MHz}) \delta: 3.81(\mathrm{~s}, 3 \mathrm{H}, \mathrm{CH} 3), 6.8(\mathrm{~m}, 1 \mathrm{H}$, $\mathrm{NH}), 7.01$ (t, 1H, J=7.82, Ar), 7.21(m, 2H, Ar), 7.36(m, $2 \mathrm{H}, \mathrm{Ar}), 7.41(\mathrm{~m}, 1 \mathrm{H}, \mathrm{Ar}), 7.59(\mathrm{~m}, 2 \mathrm{H}, \mathrm{Ar}), 8.33$ (t, 3H, $\mathrm{J}=7.76, \mathrm{Ar}), 8.59$ (dd, $1 \mathrm{H}, \mathrm{J}=7.26$, Ar). ${ }^{13} \mathrm{C}$ NMR (DMSOd6, $100 \mathrm{MHz}) \delta: 103.6,114.0,116.1,120.7,124.1,126.5$, $127.1,129.2,130.1,131.5,136.7,153.0,155.1,157.9$, 159.0. HRMS (EI) m/z calcd for $\mathrm{C}_{20} \mathrm{H}_{16} \mathrm{~N}_{4} \mathrm{O} 328.1324$, found 328.0259. Anal: C (73.15/73.34), H (4.91/5.02), N (17.06/17.13), O (4.87/4.18). 


\subsubsection{N-(3-Methoxyphenyl)-2-phenylpyrido[2,3-} d]pyrimidin-4-amine (6l)

Yield: $66 \%$. mp: $167-169^{\circ} \mathrm{C}$ (ethanol). IR (KBr) $\mathrm{cm}^{-1}: 3423.1,3376.2,3319.2,1657.4,1655.1,1619.8 .{ }^{1} \mathrm{H}$ NMR (DMSO-d6, $400 \mathrm{MHz}) \delta: 2.8(\mathrm{~s}, 3 \mathrm{H}, \mathrm{CH} 3), 6.5(\mathrm{~m}$, $2 \mathrm{H}, \mathrm{Ar}), 6.02(\mathrm{~m}, 3 \mathrm{H}, \mathrm{Ar}), 7.4(\mathrm{~s}, 2 \mathrm{H}, \mathrm{Ar}), 7.6(\mathrm{~s}, 3 \mathrm{H}, \mathrm{Ar})$, $2.5(\mathrm{~d}, 2 \mathrm{H}, \mathrm{J}=7.2, \mathrm{Ar}), 9.0(\mathrm{bs}, 1 \mathrm{H}, \mathrm{NH}) .{ }^{13} \mathrm{C}$ NMR (DMSOd6, $100 \mathrm{MHz}) \delta: 103.6,114.0,116.1,120.7,124.1,126.5$, $127.1,129.2,130.1,131.5,136.7,153.0,155.1,157.9$, 159.0. HRMS (EI) $\mathrm{m} / \mathrm{z}$ calcd for $\mathrm{C}_{20} \mathrm{H}_{16} \mathrm{~N}_{4} \mathrm{O} 328.1324$, found 328.0259. Anal: C (73.15/73.34), H (4.91/5.02), N (17.06/17.13), O (4.87/4.18).

\subsubsection{N-(2-Methoxyphenyl)-2-phenylpyrido[2,3- d]pyrimidin-4-amine (6m)}

Yield: $66 \%$ mp: $167-169^{\circ} \mathrm{C}$ (ethanol). IR $(\mathrm{KBr}) \mathrm{cm}^{-1}$ : 3423.5, 3375.3, 3318.1, 1656.3, 1656.2, 1618.7. ${ }^{1} \mathrm{H}$ NMR (DMSO-d6, $400 \mathrm{MHz}) \delta: 3.82(\mathrm{~s}, 3 \mathrm{H}, \mathrm{CH} 3), 6.8(\mathrm{~m}, 1 \mathrm{H}$, $\mathrm{NH}), 7.01(\mathrm{t}, 1 \mathrm{H}, \mathrm{J}=7.82, \mathrm{Ar}), 7.21(\mathrm{~m}, 2 \mathrm{H}, \mathrm{Ar}), 7.36(\mathrm{~m}$, 2H, Ar), 7.41(m, 1H, Ar), 7.59(m, 2H, Ar), 8.33 (t, 3H, $\mathrm{J}=7.76$, Ar), $8.59\left(\mathrm{dd}, 1 \mathrm{H}, \mathrm{J}=7.26\right.$, Ar). ${ }^{13} \mathrm{C}$ NMR (DMSO$\mathrm{d} 6,100 \mathrm{MHz}) \delta: 103.6,114.0,116.1,120.7,124.1,126.5$, $127.1,129.2,130.1,131.5,136.7,153.0,155.1,157.9$, 159.0. HRMS (EI) $\mathrm{m} / \mathrm{z}$ calcd for $\mathrm{C}_{20} \mathrm{H}_{16} \mathrm{~N}_{4} \mathrm{O} 328.1324$, found 328.0259. Anal: C (73.15/73.34), H (4.91/5.02), N (17.06/17.13), O (4.87/4.18).

\subsubsection{4-((2-Phenylpyrido[2,3-d]pyrimidin-4-yl) amino)phenol (6n)}

Yield: $65 \%$. mp: $130-132^{\circ} \mathrm{C}$ (ethanol). IR $(\mathrm{KBr}) \mathrm{cm}^{-1}$ : 3612.1, 3423.6, 3376.3, 3319.3, 1657.6, 1655.5, 1619.7. ${ }^{1} \mathrm{H}$ NMR (DMSO-d6, $\left.400 \mathrm{MHz}\right) \delta: 6.7(\mathrm{~m}, 2 \mathrm{H}, \mathrm{Ar})$, 7.0(m, 2H, Ar), 7.21(m, 2H, Ar), 7.85(t, 2H, J=7.8, Ar), 7.9(m, 2H, Ar), 8.30(m, 2H, Ar), 8.91(d, 2H, J=7.75, Ar), 10.89(s, 1H, Ar-OH). ${ }^{13} \mathrm{C}$ NMR (DMSO-d6, $100 \mathrm{MHz}$ ) $\delta: 103.6,114.0,116.1,120.7,124.1,126.5,127.1,129.2$, $130.1,131.5,136.7,153.0,155.1,157.9,159.0$. HRMS (EI) $\mathrm{m} / \mathrm{z}$ calcd for $\mathrm{C}_{19} \mathrm{H}_{14} \mathrm{~N}_{4} \mathrm{O} 314.1168$, found 314.1100 . Anal: C (72.60/72.69), H (4.49/4.56), N (17.85/17.67), O $(5.09 / 5.11)$.

\subsubsection{2-((2-Phenylpyrido[2,3-d]pyrimi- din-4-yl)amino)phenol (6o)}

Yield: $67 \%$. mp: $129-131^{\circ} \mathrm{C}$ (ethanol). IR (KBr) $\mathrm{cm}^{-1}$ : 3651.5, 3423.5, 3378.2, 3319.4, 1657.5, 1655.5, 1619.7. ${ }^{1} \mathrm{H}$ NMR (DMSO-d6, $\left.400 \mathrm{MHz}\right) \delta: 7.2(\mathrm{~m}, 7 \mathrm{H}, \mathrm{Ar}), 7.32(\mathrm{t}$, $2 \mathrm{H}, \mathrm{J}=7.58, \operatorname{Ar}), 7.5(\mathrm{~m}, 2 \mathrm{H}, \mathrm{Ar}), 8.0(\mathrm{~d}, 2 \mathrm{H}, \mathrm{J}=7.8, \mathrm{Ar})$, 12.52(bs, $1 \mathrm{H}, \mathrm{Ar}-\mathrm{OH}) .{ }^{13} \mathrm{C}$ NMR (DMSO-d6, $100 \mathrm{MHz}$ ) $\delta: 103.6,114.0,116.1,120.7,124.1,126.5,127.1,129.2$, 130.1, 131.5, 136.7, 153.0, 155.1, 157.9, 159.0. HRMS (EI) $\mathrm{m} / \mathrm{z}$ calcd for $\mathrm{C}_{19} \mathrm{H}_{14} \mathrm{~N}_{4} \mathrm{O} 314.1168$, found 314.1110 . Anal: C (72.60/72.65), H (4.49/4.57), N (17.85/17.69), O $(5.09 / 5.10)$.

\subsubsection{N-(4-Nitrophenyl)-2-phenylpyrido[2,3-d] pyrimidin-4-amine (6p)}

Yield: $66 \%$ mp: $172-174^{\circ} \mathrm{C}$ (ethanol). IR $(\mathrm{KBr}) \mathrm{cm}^{-1}$ : 3423.1, 3376.2, 3319.7, 1658.4, 1655.1, 1619.1. ${ }^{1} \mathrm{H}$ NMR (DMSO-d6, $400 \mathrm{MHz}) \delta:$ 6.89(bs, 1H, NH), 7.44(m, 4H, Ar), 7.50 (m, 3H, Ar), 8.03(d, 2H, J=7.16, Ar), 8.36(t, 2H, $\mathrm{J}=7.32$, Ar), 8.59(t, $1 \mathrm{H}, \mathrm{J}=7.75$, Ar). ${ }^{13} \mathrm{C}$ NMR (DMSOd6, $100 \mathrm{MHz}) \delta: 103.6,114.0,116.1,120.7,124.1,126.5$, $127.1,129.2,130.1,131.5,136.7,153.0,155.1,157.9$, 159.0. HRMS (EI) $\mathrm{m} / \mathrm{z}$ calcd for $\mathrm{C}_{19} \mathrm{H}_{13} \mathrm{~N}_{5} \mathrm{O}_{2} 343.1069$, found 343.1259. Anal: $\mathrm{C}(66.47 / 66.32), \mathrm{H}(3.82 / 3.90), \mathrm{N}$ (20.40/20.67), O (9.32/9.44).

\subsubsection{N-(3-Nitrophenyl)-2-phenylpyrido[2,3-d] pyrimidin-4-amine (6q)}

Yield: $60 \%$ mp: $177-179^{\circ} \mathrm{C}$ (ethanol). IR $(\mathrm{KBr}) \mathrm{cm}^{-1}$ : 3425.2, 3377.1, 3318.1, 1657.7, 1655.8, 1619.2. ${ }^{1} \mathrm{H}$ NMR (DMSO-d6, $400 \mathrm{MHz}) \delta: 6.89(\mathrm{bs}, 1 \mathrm{H}, \mathrm{NH}), 7.2(\mathrm{~m}, 1 \mathrm{H}$, Ar), 7.44(m, 3H, Ar), 7.50(m, 3H, Ar), 8.03(d, 2H, J=7.16, Ar), 8.36(t, 2H, J=7.32, Ar), 8.59(t, $1 \mathrm{H}, \mathrm{J}=7.75, \mathrm{Ar}) .{ }^{13} \mathrm{C}$ NMR (DMSO-d6, 100 MHz) $\delta: 103.6,114.0,116.1,120.7$, $124.1,126.5,127.1,129.2,130.1,131.5,136.7,153.0$, 155.1, 157.9, 159.0. HRMS (EI) $\mathrm{m} / \mathrm{z}$ calcd for $\mathrm{C}_{19} \mathrm{H}_{13} \mathrm{~N}_{5} \mathrm{O}_{2}$ 343.1069, found 343.1123. Anal: $\mathrm{C}(66.47 / 66.34), \mathrm{H}$ (3.82/3.95), N (20.40/20.45), O (9.32/9.40).

\subsubsection{N-(2-Nitrophenyl)-2-phenylpyrido[2,3-d] pyrimidin-4-amine (6r)}

Yield: $65 \%$. mp: $172-174{ }^{\circ} \mathrm{C}$ (ethanol). IR (KBr) $\mathrm{cm}^{-1}$ : 3423.9, 3376.2, 3319.5, 1657.1, 1656.6, 1617.2. ${ }^{1} \mathrm{H}$ NMR (DMSO-d6, $\left.400 \mathrm{MHz}\right) \delta: 6.89$ (bs, 1H, NH), 7.2 (m, 1H, Ar), 7.44 (m, 3H, Ar), 7.50 (m, 3H, Ar), 8.03 $(\mathrm{d}, 2 \mathrm{H}, \mathrm{J}=7.16, \mathrm{Ar}), 8.36(\mathrm{t}, 2 \mathrm{H}, \mathrm{J}=7.32, \mathrm{Ar}), 8.59(\mathrm{t}, 1 \mathrm{H}$, $\mathrm{J}=7.75$, Ar). ${ }^{13} \mathrm{C}$ NMR (DMSO-d6, $\left.100 \mathrm{MHz}\right) \delta: 103.6$, $114.0,116.1,120.7,124.1,126.5,127.1,129.2,130.1$, $131.5,136.7,153.0,155.1,157.9,159.0$. HRMS (EI) $\mathrm{m} / \mathrm{z}$ calcd for $\mathrm{C}_{19} \mathrm{H}_{13} \mathrm{~N}_{5} \mathrm{O}_{2}$ 343.1060, found 343.1089 . Anal: C (66.47/66.32), H (3.82/3.94), N (20.40/20.44), O (9.32/9.39).

\subsection{Biological Studies}

\subsubsection{Kinetic Assays}

\section{Chemicals and enzymes}

The enzyme biotin carboxylase was isolated from Escherichia coli by gene overexpression method as reported elsewhere. ${ }^{[28]}$ The obtained enzyme was purified using the nickel affinity chromatography by histidine-tag attached to $\mathrm{N}$-terminal of the enzyme. ${ }^{[29]}$ Bradford method was used to determine the concentration of enzyme using bovine serum albumin as reference standard. ${ }^{[30]}$ All other reagents, intermediates and solvents were procured from Sigma Aldrich. 


\section{Biotin carboxylase assay}

The enzyme activity of biotin carboxylase was measured by continuous spectrophotometric method. ADP production was detected by coupling of enzymes pyruvate kinase and lactate dehydrogenase as described earlier. ${ }^{[30]}$ Theeffect of synthetic compounds on the activity of coupling enzymes was measured at $340 \mathrm{mM}$. All the experiments were conducted in variable concentrations and by measuring the inhibition of the enzyme activity. The reactions were carried out at a total of $0.5 \mathrm{~mL}$ of solution in quartz cuvette with $1 \mathrm{~cm}$ path. Before every measurement, the reactions were initiated by the addition of enzyme. All the spectrophotometric experiments were carried out on a Shimadzu UV-vis spectrophotometer with data acquisition software.

\section{Data analysis}

The data obtained from the enzyme inhibition assay were analysed by the nonlinear regression method by application of computer program developed by Cleland. ${ }^{[31]}$ The data obtained was fitted in to the equation mentioned below;

$$
\begin{gathered}
v=\frac{V A}{K m\left(1+\frac{I}{K i s}\right)+A} \\
v=\frac{V A}{K m\left(1+\frac{I}{K i s}\right)+A\left(1+\frac{I}{K i i}\right)}
\end{gathered}
$$

Where,

$\mathrm{v}=$ initial velocity

$\mathrm{V}=$ maximal velocity

$\mathrm{A}=$ substrate concentration

$\mathrm{Km}=$ Michalis constant

$\mathrm{I}=$ concentration of the inhibitor

Kis and $\mathrm{Kii}=$ slopes and intercept inhibition constants.

\subsubsection{Determination of MICs.}

Minimum Inhibitory concentration MICs were determined by the broth microdilution methods using a cation-adjusted Mueller Hinton (MHII) medium. ${ }^{[32]}$ The strains of microorganism were obtained from the Institute of Microbial Technology (IMTEC), Chandigarh and the ATCC numbers are mentioned accordingly. Agar medium was streaked with test microorganism and incubated overnight at $35^{\circ} \mathrm{C}$. The test compounds were prepared over a serial dilution method, by solubilizing compounds in 100\% DMSO and sterile water for standard drugs gentamicin and kanamycin to attain a final stock concentration of $10 \mathrm{mg} / \mathrm{L}$. The inoculum of $5 \times 105 \mathrm{CFU} / \mathrm{mL} / 0.1 \mathrm{~mL}$ in standardized form was incubated with antibiotic for $18-24 \mathrm{~h}$ at $35^{\circ} \mathrm{C}$.
Visual measures were used to record the MICs and scored accordingly and lowest concentration of antibiotic and the test compounds where observed for the visual inspection for turbidity.

\subsubsection{Generation of Spontaneous Resistant Mutant Strains and Resistance Studies.}

MHII agar was molten and cooled to $55{ }^{\circ} \mathrm{C}$, to this compounds $6 \mathrm{~d}, 61,6 \mathrm{n}, 6 \mathrm{o}$ were added in a concentration of two-fold increase starting with initial MIC observed from the previous experiment. Single-step spontaneous resistance selected cultures were obtained as described elsewhere. The microorganisms were allowed to grow overnight to saturation in MHII broth. The microbial cells were concentrated by centrifugation of the medium and a viable number of colony forming units (CFU) were determined by serial dilutions and plating technique. It was taken care that each individual plate had around $4 \times 109 \mathrm{CFU} /$ plate. Subsequently the culture plates were monitored for the development of drug-resistant colonies and were observed visually. A detailed study on the development of moiramide resistance and further evaluation was performed as per the earlier reported procedure. ${ }^{[33]}$

\subsubsection{Molecular Docking Studies}

All the in silico designing and docking studies were performed on the Schrodinger modelling suite (Version 15.3), the compounds designed were subjected to energy minimisation by Ligprep module of the software. ${ }^{[34]}$ All the protein preparation work was carried out on the protein preparation wizard of the Glide module, the crystal structure obtained from the protein data bank contains the ligands, cofactors, water molecules, they may also be deficient of certain loops, and these anomalies are refined by the protein preparation wizard. The cofactor unless essential are removed the ligand is replaced and the water molecules are also eliminated. The missing loops are filled and the caps are inserted. Thus, when the protein is ready energy minimisation program is run to optimise the protein for molecular docking studies. Glide module was employed for the molecular docking; compounds were docked in the grid designed in the module. The docking was carried out with flexible ligands and the protein with the simulations made on the OPLS5.0 force field. All the results were based on 10 conformations for each molecule in the extra precision (XP) mode. The crystal structure (PDB 2V58) ${ }^{[8]}$ was employed as the receptor with compounds 6a-r as ligands. The results obtained from the simulation were obtained in the form of dock score; these values represent the minimum energies. Interactions between the ligand and residues were presented in the form of H-bond, van der Waals forces and the pi bonds. The results in the form of 3D and 2D representation were obtained for simplified understanding. 


\section{RESULTS AND DISCUSSION}

Biotin carboxylase acts as an important catalyst in the synthesis of malonyl-CoA which is an important precursor for the synthesis of fatty acids. These are essential for formation of the bacterial cell wall. With the advent of drug resistant bacterial strains and urgent need for drugs with potential for inhibiting drug resistant bacteria was felt. Herein, we are reporting the development of some new pyridopyrimidine derivatives with their potential for inhibiting the drug resistant bacterial strains. Earlier, Chakravarty et al., has reported several quinazolines as TGF- $\beta$ inhibitors. ${ }^{[35]}$ Pyridopyrimidines were designed on the basis of structural analogy to the compounds previously reported with antibacterial potential; these consisted of some common features like the pyridopyrimidine nucleus as shown in (Figure 2). $\left[{ }^{8,14,22-24]}\right.$ Eighteen pyridopyrimidine derivatives were synthesised following a five-step synthetic protocol as shown in scheme 1 .<smiles>Nc1ncccc1C(=O)O</smiles>

1 (i)<smiles>COC(=O)c1cccnc1N</smiles>

(ii)<smiles>CC#CC</smiles><smiles>COC(=O)c1cccnc1N</smiles><smiles>NC(=O)c1ccccc1</smiles>

(iii)<smiles>[R]Nc1nc(-c2ccccc2)nc2ncccc12</smiles>

(v)<smiles>Clc1nc(-c2ccccc2)nc2ncccc12</smiles>
5<smiles>O=c1[nH]c(-c2ccccc2)nc2ncccc12</smiles>

Scheme 1: Synthesis of pyridopyrimidine derivatives. Reagents and conditions: (i) $\mathrm{SOCl} 2, \mathrm{CH} 3 \mathrm{OH}$, (ii) 3-Chlorobenzoyl chloride, $\mathrm{CHCl}$, (C2H5)3N, (iii) $2 \mathrm{M} \mathrm{NH} 3 / \mathrm{CH} 3 \mathrm{OH}, 28 \% \mathrm{NH} 4 \mathrm{OH}$ (iv) $\mathrm{POCl} 3$ (v) (C2H5)3N, DMF.

2-Amino pyridine carboxylic acid (1) undergoes Fischer-Speier esterification[36] and provides with methyl 2-aminonicotinate (2). The free primary amine reacts with 3-chlorobenzoyl chloride in the presence of triethyl amine and forms the methyl 2-benzamidonicotinate (3). Compound (3) undergoes cyclisation in the presence of ammonia leading to formation of 2-phenylpyrido[2,3d]pyrimidin-4(3H)-one (4). On treatment with phosphorus oxychloride, compound (4) forms the 4-chloro-2-phenylpyrido[2,3-d]pyrimidine (5). The chloro group of compound five is substituted with various anilines in basic medium to give eighteen final derivatives. The structural confirmation was made on the basis of IR, NMR and mass spectra.

The spectral details of compounds are mentioned in the experimental section with spectra of selected compounds in the supplementary material available with the online version of this article. Compound (2) shows the characteristic amine absorption spectra around $3424.1 \mathrm{~cm}^{-1}$ and the absorption for ester around $1655.1 \mathrm{~cm}^{-1}$. Proton NMR spectra of this compound shows a broad singlet corresponding to the amine at $12.19 \mathrm{ppm}$ and a singlet at 3.86 ppm with three protons of the methyl group. The compound (3) shows absorption around $1455.1 \mathrm{~cm}^{-1}$ for the secondary amine group, the proton NMR spectra shows a singlet of the methyl group at $3.77 \mathrm{ppm}$ with three protons, aromatic protons around 7.7 to $8.3 \mathrm{ppm}$ whereas the amide proton is seen at $12.73 \mathrm{ppm}$. In case of compound (4) the methyl group seen in compound (3) disappears as a result of cyclisation and a single proton of secondary nitrogen is observed at $5.8 \mathrm{ppm}$ as abroad singlet. 
The compound (5) shows all the protons in the aromatic region because chlorination of compound (4) leads to disappearance of amide proton. In the series of compounds (6a-r), the characteristic properties of derivatives involved singlets at $2.4 \mathrm{ppm}$ corresponding to the methyl group of the tolyl derivative (6i). singlet at $2.12 \mathrm{ppm}$ of methyl group of the tolyl derivative $(6 \mathrm{j})$. The methoxy derivatives (6k), (61) and (6m) showed a methyl proton at 3.81, 2.8 and $3.82 \mathrm{ppm}$ respectively. In case of compounds (6n) and (6o) showed characteristic-OH broad singlets at 10.89 and $12.52 \mathrm{ppm}$ respectively. All the compounds displayed the number of carbon atoms as per the molecular formula and the characteristic signals were observed for the compounds like in (2) the carboxyl carbon signals at $167.5 \mathrm{ppm}$, in compound (3) there are two carboxyl carbons at 167.1 and 168.1 respectively, in compound (4) the amide carbons show signal at $161.0 \mathrm{ppm}$. All compounds were subjected to determination of molecular weight following a mass spectral analysis; all compounds were found to be in agreement with the theoretical values.
The synthesised compounds were then subjected to enzyme kinetics assay using the biotin carboxylase enzyme obtained as per the protocol mentioned in the experimental section. Compounds were tested against the $E$. Coli of wild type and tolC mutant resistant stain, $P$. aeruginosa wild type and $\delta$-RND mutant resistant variety, $S$. aureus wild and Moiramide resistant mutant strain, $S$. epidermidis wild type and E. faecalis wild type strains. All the compounds were subjected to the assay in varying concentrations in the reaction mixture consisting of $\mathrm{BC}$ and other reagents (Figure 3). The UV absorptions of these compounds were recorded in the computer acquisition system and the readings were calculated using kinetic analysis formula provided by Cleland. It was observed that compounds $6 \mathrm{~d}, 61$, $6 \mathrm{n}$ and $6 \mathrm{o}$ shows non-competitive inhibition with ATP and showed the $\mathrm{K}_{\mathrm{is}}$ around $29.8 \pm 12.8 \mathrm{nM}$ and a $\mathrm{K}_{\mathrm{ii}}$ of $33.8 \pm$ $7.4 \mathrm{nM}$. The inhibitory concentrations for compounds $6 \mathrm{a}-\mathrm{r}$ is presented in (Table 1).

Table 1: Antibacterial activity of synthesised compounds

$\operatorname{MIC}(\mu \mathrm{g} / \mathrm{mL})$

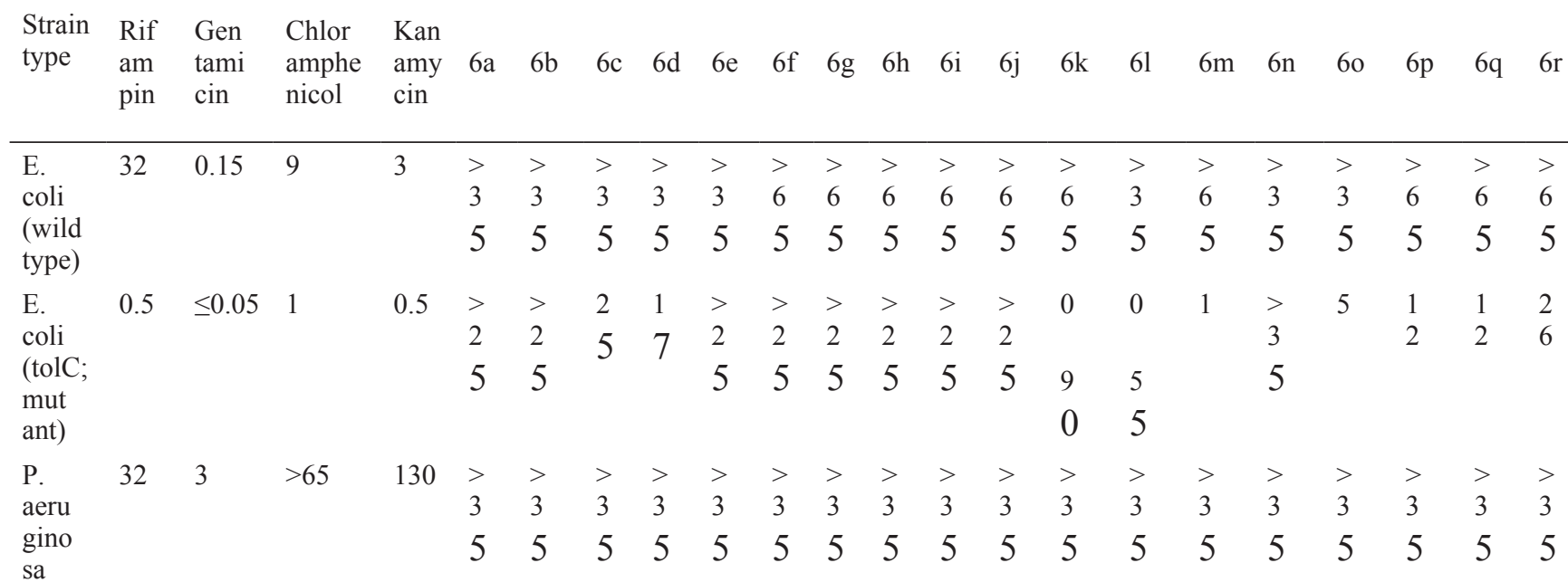

(wil

type)

$\begin{array}{llllllllllllllllllllllllll}\text { P. } & 32 & 0.7 & 3 & 65 & > & 9 & > & > & > & > & > & > & > & > & 5 & 9 & 1 & > & > & > & > & > \\ \text { aeru } & & & & & 3 & & 3 & 3 & 3 & 3 & 3 & 3 & 3 & 3 & 1 & & 7 & 3 & 3 & 3 & 3 & 3 \\ \text { gino } & & & & & 5 & & 5 & 5 & 5 & 5 & 5 & 5 & 5 & 5 & & & & 5 & 5 & 5 & 5 & 5\end{array}$

$\mathrm{RN}$

$\mathrm{D}$

mut

ant) S.

$\leq 0 . \quad 0.6 \quad 9$

(wil

d

type) 


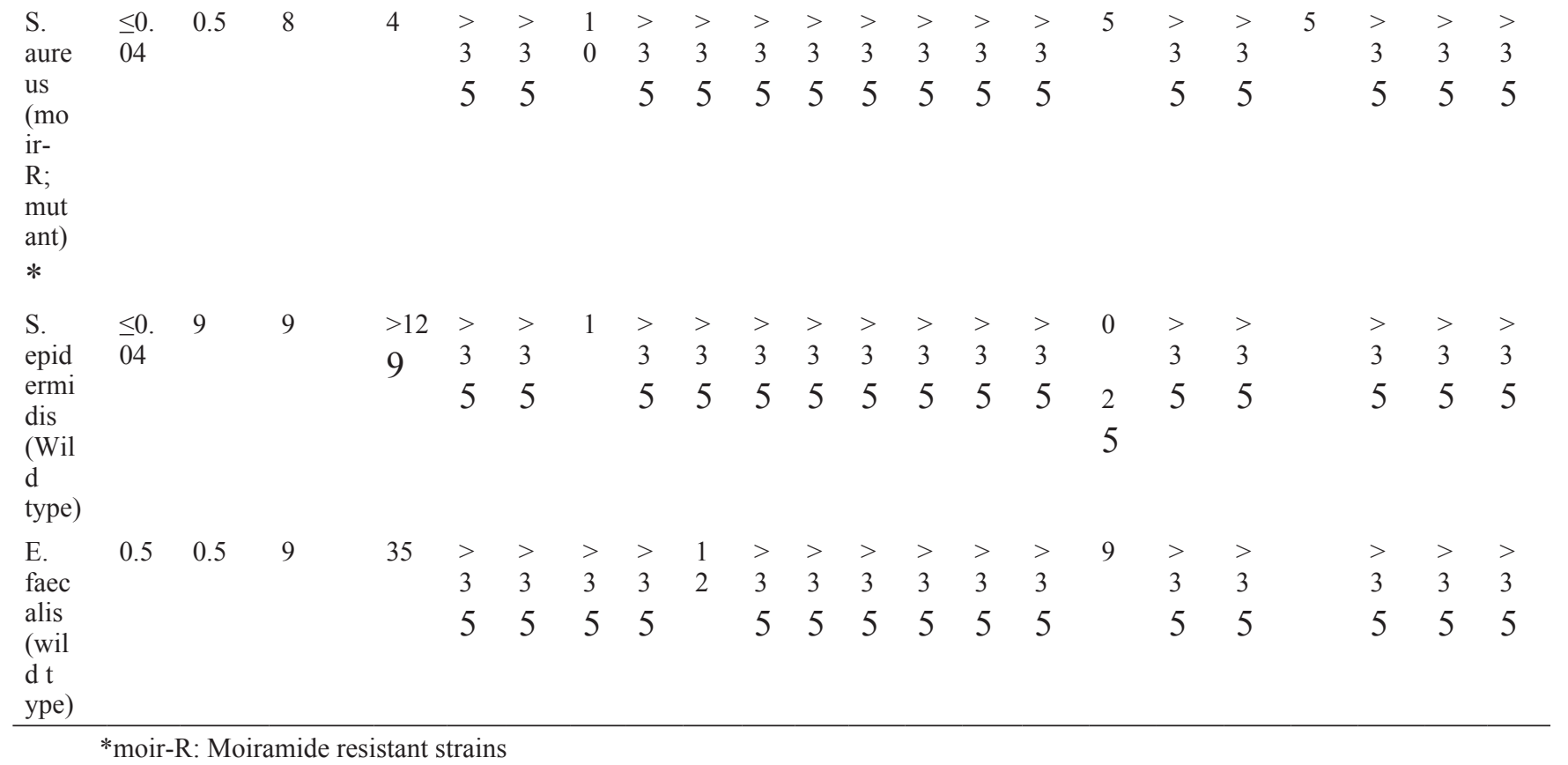

*moir-R: Moiramide resistant strains

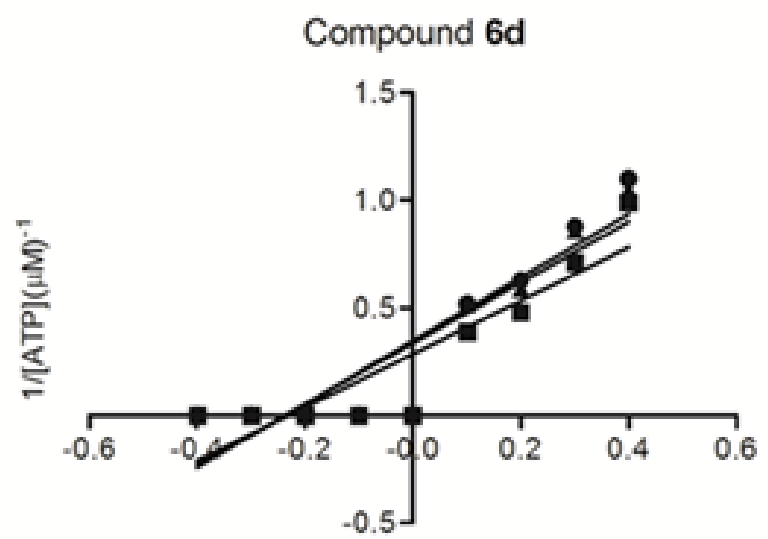

$1 / \mathrm{v}(\mu \mathrm{mol} / \mathrm{min} / \mathrm{mg})^{-1}$

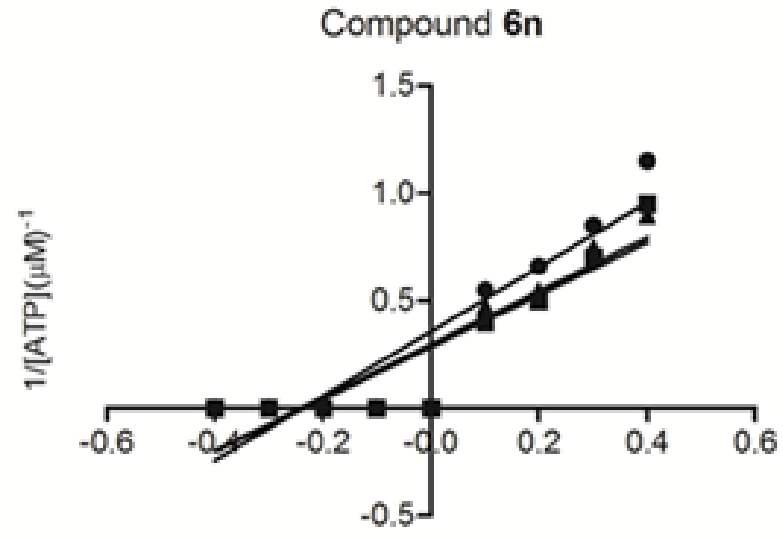

$1 / \mathrm{V}(\mu \mathrm{mol} / \mathrm{min} / \mathrm{mg})^{-1}$

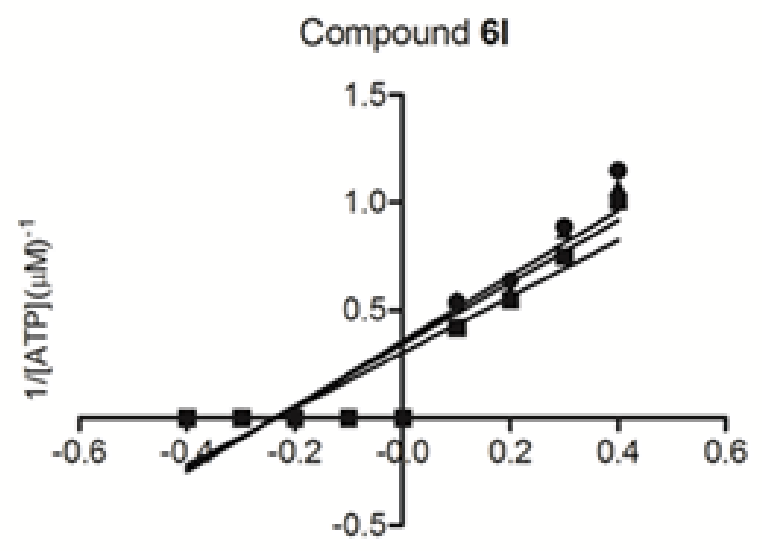

$1 / \mathrm{v}(\mu \mathrm{mol} / \mathrm{min} / \mathrm{mg})^{-1}$

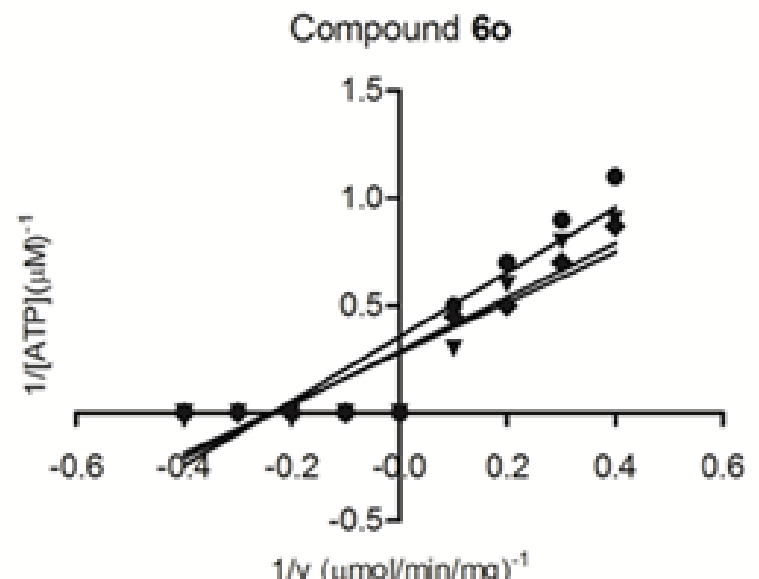

Fig. 3: Compound 6d: Inhibition at $0(\bullet), 0.1(\boldsymbol{\bullet}), 0.2(\boldsymbol{\Delta})$ and $0.3(\diamond)$ nM. Compound 61: Inhibition at $0(\bullet), 0.1(\boldsymbol{\bullet}), 0.2(\boldsymbol{\Delta})$ and $0.3(\bullet)$ nM. Compound 6n: Inhibition at $0(\bullet), 0.1(\bullet), 0.2(\boldsymbol{\Delta})$ and $0.3(\bullet) \mathrm{nM}$. Compound 6o: Inhibition at $0(\bullet), 0.1(\boldsymbol{\bullet}), 0.2(\boldsymbol{\Delta})$ and $0.3(\bullet) \mathrm{nM}$. The substrate ATP was varied, HCO3- and acetyl-CoA was held constant. 
The compound $6 \mathrm{~d}, 61,6 \mathrm{n}$ and 60 were selected from the kinetic assay for determination of their antibacterial activity. The Minimum Inhibitory Concentrations (MICs) were evaluated following the standard protocol, the details are mentioned in experimental section and results are given in table 1 . Compound $6 \mathrm{~d}$ exhibited antibacterial activity against mutant type $E$. coli at $17 \mu \mathrm{g} / \mathrm{mL}$, which is higher compared to standard drug used in therapy. Compound 61 exhibited comparative antibacterial activity against mutant type of E. coli at $0.55 \mu \mathrm{g} / \mathrm{mL}$ which is similar to rifampin and kanamycin. Compound 61 showed inhibitory activity at concentration of $9 \mu \mathrm{g} / \mathrm{mL}$ against P.aeruginosa better than rifampin. Compounds 61 and 60 exhibited comparative inhibition of wild type and mutant type of S. aureus. Compound 61 showed a good inhibitory activity against $S$. epidermidis at $0.25 \mu \mathrm{g} / \mathrm{mL}$ and $\mathrm{E}$. faecalis at $9 \mu \mathrm{g} / \mathrm{mL}$. Compound $6 \mathrm{o}$ showed good activity against wild and mutant type of $S$. aureus at 4 and $5 \mu \mathrm{g} / \mathrm{mL}$ respectively, it exhibited superior activity against wild type of $S$. epidermidis and wild type of E. faecalis at 2 and $5 \mu \mathrm{g} / \mathrm{mL}$ respectively. Thus, the MICs of compounds showed a comparative activity against the wild and mutant types of E. coli, $P$. aeruginosa, S. aureus, S. epidermidis and E. faecalis. The details of MIC for remaining compounds are provided in the supplementary information (Table S2).

Table 2: Single step resistance studies on E. Coli

\begin{tabular}{|c|c|c|c|c|}
\hline Compound & $\mathrm{MIC}(\mu \mathrm{g} / \mathrm{mL})$ & $\mathrm{MPC} \mu \mathrm{g} / \mathrm{mL}$ & MSW & Frequency of resistance \\
\hline Rifampin & 8 & $>64$ & $>8$ & $7 \times 10^{-9}$ \\
\hline 61 & 1 & 32 & 32 & $<3 \times 10^{-9}$ \\
\hline $6 n$ & 16 & 84 & $>5$ & $<3 \times 10^{-9}$ \\
\hline 60 & 16 & 84 & $>5$ & $<3 \times 10^{-9}$ \\
\hline
\end{tabular}

MIC: minimum inhibitory concentration,

MPC: mutant prevention concentration,

MSW: mutant selection windows

To determine the potential of these compounds we carried out an experiment to determine the single step resistance study and frequency of developing resistance for three compounds $61,6 \mathrm{n}$ and 60 with rifampin as a reference standard. Compound $6 \mathrm{n}$ and 60 was found to have higher mutant prevention concentration (MPC) at $84 \mu \mathrm{g} /$ $\mathrm{mL}$ and compound 61 showed the MPC of $32 \mu \mathrm{g} / \mathrm{mL}$. The MPC dose of drug at which resistant mutant strain were no longer obtained after using 4 x $109 \mathrm{CFU}$. The mutant selection windows (MSWs) is a ratio of MPC: MIC, it was determined for the three compounds under study. It was found that MSW values for compound 61 was 32, for compound $6 \mathrm{n}$ and 60 it was $>5$. Compound $6 \mathrm{n}$ and 60 showed better MSW compared to compound 61 . The results suggest that compounds $6 \mathrm{n}$ and $6 \mathrm{o}$ have low potential to develop spontaneous resistance.

All the compounds were subjected to molecular docking studies to determine the mode of binding and energy changes. The crystal structure of biotin carboxylase (PDB: 2V58) was used for this study ${ }^{[34]}$ and ligands were prepared for docking by method described elsewhere. The biotin carboxylase is co-crystallised with 6-(2,6-dibromophenyl) pyrido[2,3-d]pyrimidine-2,7-diamine, which we have used as reference ligand for molecular docking studies. It was observed that compound $6 \mathrm{~d}, 61,6 \mathrm{n}$ and 60 showed highest interaction with the enzyme in their molecular docking studies, the details of docking studies are mentioned in the table S2 (Supplementary material). The bound ligand in the active site forms covalent nitration with Glu201A, Lys202A, aromatic interactions with Leu204A and Lys159A, it shows weaker interactions with Ile437A and His438. The docked compound 6d, show a high docking score of -7.58 and Glide energy of -44.50 . It forms a salt bridge between pyridopyridine nucleus and HIS438 (Figure $4 \mathrm{a}$ and $\mathrm{b}$ ). It forms an aromatic interaction with the Lys159, the presence of electronegative substituent like chlorine on the phenylpyridopyrimidine nucleus contributes to its biological activity. Compound 61, showed a high docking score of -7.34 and Glide energy of -45.06 , the pyridopyridine nucleus forms a salt bridge with the residue HIS430 and the phenyl ring formed a salt bridge with HIS209 (Figure 4c and d), this may be due to the presence of electron donating group like methoxy on the phenylpyridopyrimidine nucleus. Compound $6 \mathrm{n}$, show a docking score of -7.76 and Glide energy of -47.34. It forms a salt bridge between pyridopyridine nucleus and HIS438, the phenyl ring of substituent forms another salt bridge with HIS209 and a hydrogen bond is formed between hydroxy group with GLU276 (Figure 5a and b). Compound 6o, showed a high docking score of -7.96 and Glide energy of -46.71 , the pyridopyridine nucleus forms a salt bridge with the residue HIS438 and the $\mathrm{OH}$ group forms a hydrogen bond with GLY165 (Figure 5c and d). In case of compounds 61 and $6 n$ there is hydroxyl group $(-\mathrm{OH})$ on the $4^{\text {th }}$ and 2 nd positions respectively. Their biological activity, high dock score and hydrogen bond formation with Glu276 and Gly165 suggest importance of strong electron donating character of hydroxyl functional group on the phenylpyridopyrimidine nucleus. 


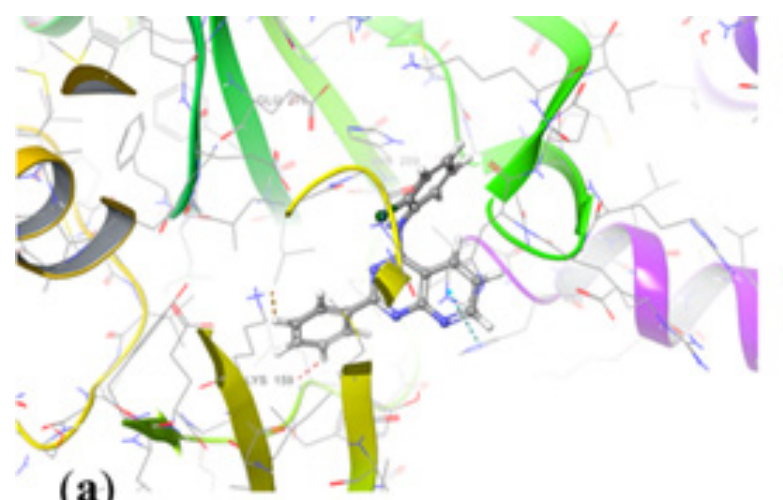

(a)
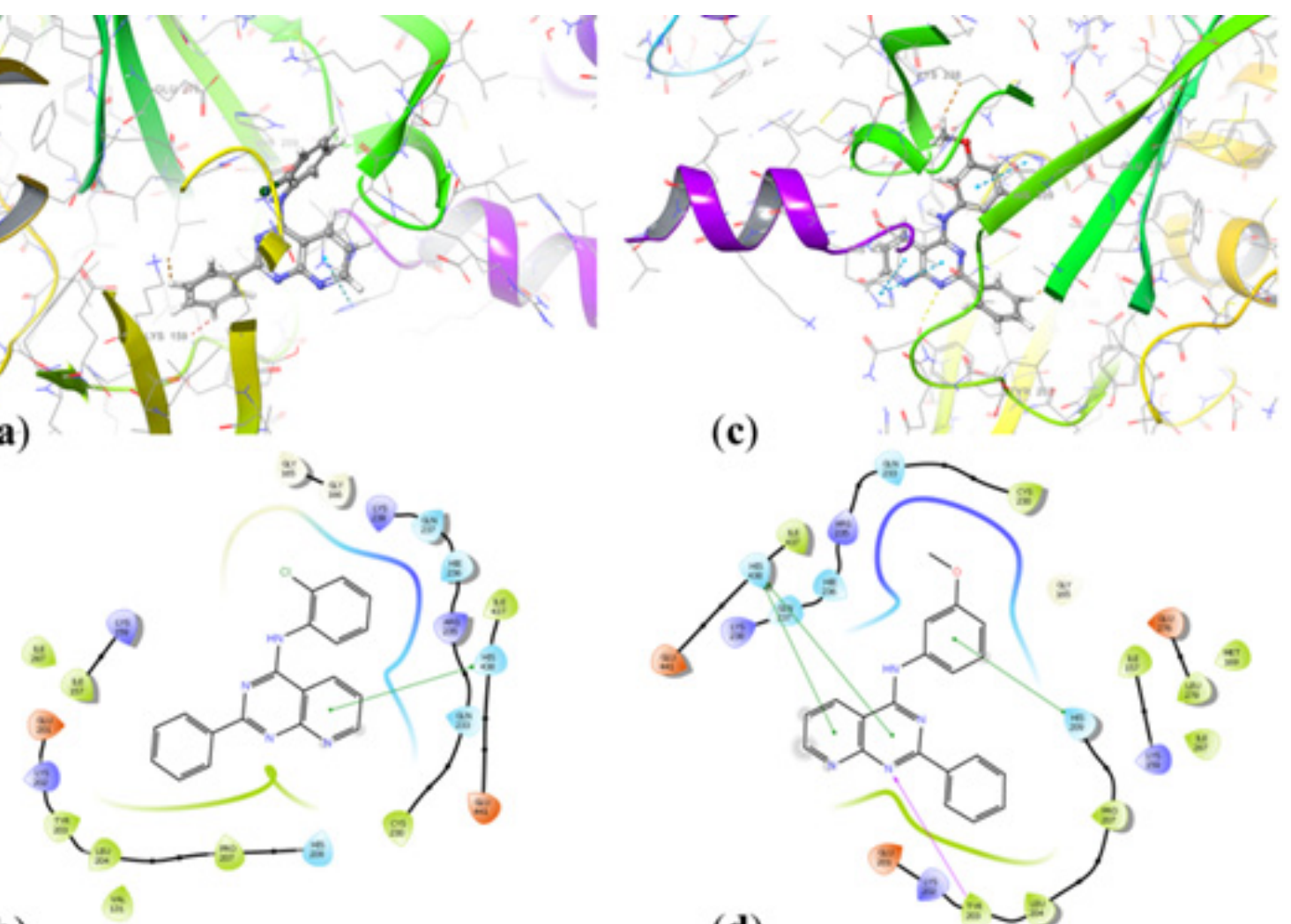

(b)

(5)

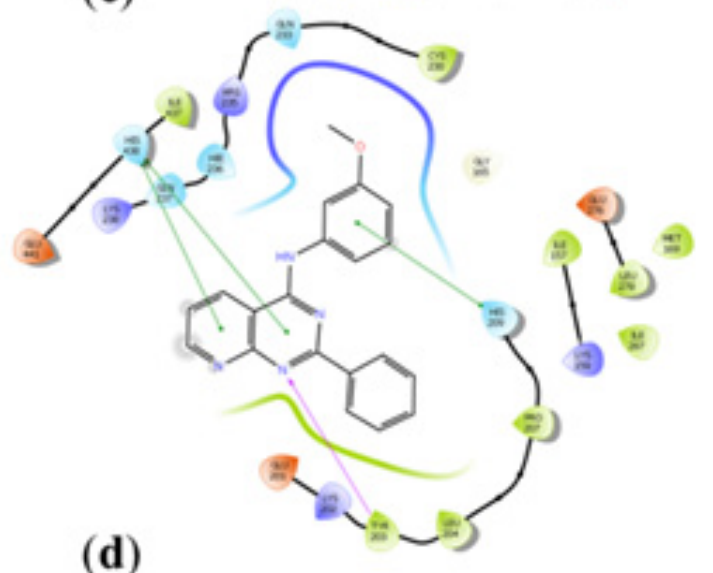

Fig. 4: (a, b) Molecular interaction between the biotin carboxylase and compound 6d, (c, d) Molecular interaction between the biotin carboxylase and compound 61

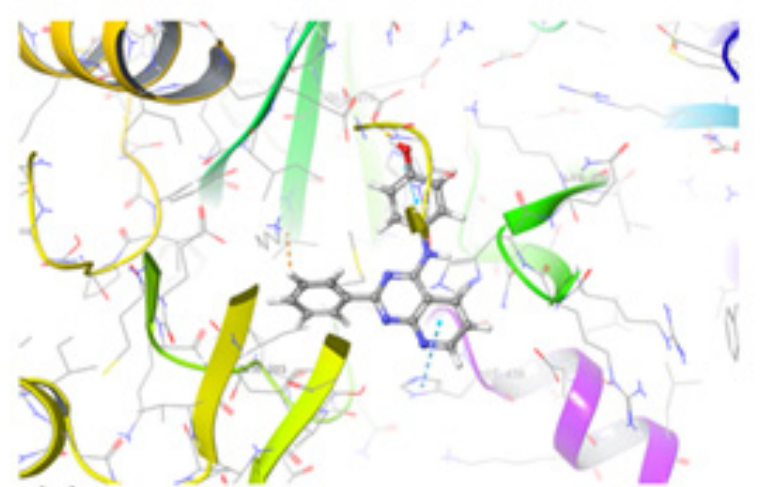

(a)
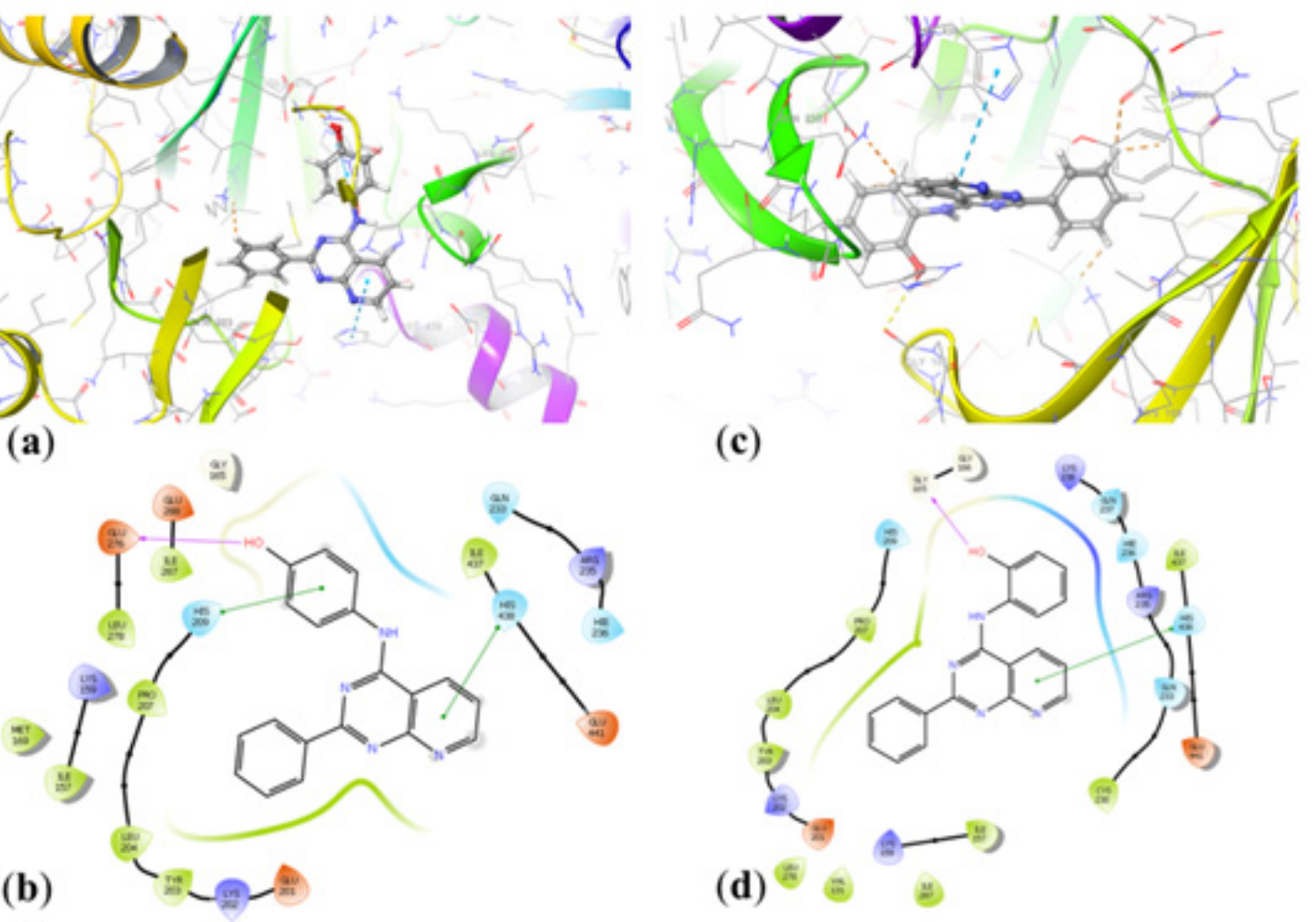

Fig. 5: (a, b) Molecular interaction between the biotin carboxylase and compound 6n, (c, d) Molecular interaction between the biotin carboxylase and compound 60 


\section{CONCLUSION}

$\mathrm{BC}$ is an important intermediate in the synthesis of malonyl CoA. Present study reports the development of novel pyridopyrimidine derivatives with potent antibacterial activity with less possibility for developing of resistance by targeting this biotin carboxylase enzyme. The series of pyridopyrimidines yielded eighteen compounds. These compounds were characterized on the basis of spectral data and further tested for their antibacterial potential by the enzyme kinetic assay against the $\mathrm{BC}$ and then MIC. Compounds 6d, 61, 6n and 6o were showed promising activity. These compounds were then tested for the single step resistance study; compound 60 was found to be a promising lead in the development of antibacterial with greater potential and lesser possibility for bacteria to be resistant to this compound. This compound can be studied further and developed into a potential antibacterial lead molecule.

\section{ACKNOWLEDGEMENTS}

VBP acknowledges the School of Pharmacy, Swami Ramanand Teerth Marathwada University for all the research facilities provided.

\section{CONFLICT OF INTEREST}

There are no conflicts of interest.

\section{REFERENCES}

1. W.H. Organization, Antimicrobial resistance: global report on surveillance, World Health Organization.

2. D.J. Payne, M.N. Gwynn, D.J. Holmes, D.L. Pompliano, Drugs for bad bugs: confronting the challenges of antibacterial discovery, Nature reviews Drug discovery 6(1) (2007) 29-40.

3. J. Clardy, M.A. Fischbach, C.R. Currie, The natural history of antibiotics, Current biology 19(11) (2009) R437-R441.

4. M.A. Kohanski, D.J. Dwyer, J.J. Collins, How antibiotics kill bacteria: from targets to networks, Nature Reviews Microbiology 8(6) (2010) 423-435.

5. Y.M. Amgaokar, R.V. Chikhale, U.B. Lade, D.M. Biyani, M.J. Umekar, Design, formulation and evaluation of transdermal drug delivery system of budesonide, Digest Journal of Nanomaterials \& Biostructures (DJNB) 6(2) (2011).

6. U.B. Lade, Y.M. Amgaonkar, R.V. Chikhale, D.M. Biyani, M.J. Umekar, Design, formulation and evaluation of transdermal drug delivery system of budesonide, Pharmacology \& Pharmacy 2(03) (2011) 199.
7. E. Choi-Rhee, J.E. Cronan, The biotin carboxylasebiotin carboxyl carrier protein complex of Escherichia coli acetyl-CoA carboxylase, Journal of Biological Chemistry 278(33) (2003) 30806-30812.

8. J.R. Miller, S. Dunham, I. Mochalkin, C. Banotai, M. Bowman, S. Buist, B. Dunkle, D. Hanna, H.J. Harwood, M.D. Huband, A class of selective antibacterials derived from a protein kinase inhibitor pharmacophore, Proceedings of the National Academy of Sciences 106(6) (2009) 1737-1742.

9. I. Mochalkin, J.R. Miller, L. Narasimhan, V. Thanabal, P. Erdman, P.B. Cox, J.V. Prasad, S. Lightle, M.D. Huband, C.K. Stover, Discovery of antibacterial biotin carboxylase inhibitors by virtual screening and fragment-based approaches, ACS chemical biology 4(6) (2009) 473-483.

10. R. Chikhale, S. Menghani, R. Babu, R. Bansode, G. Bhargavi, N. Karodia, M. Rajasekharan, A. Paradkar, P. Khedekar, Development of selective DprE1 inhibitors: Design, synthesis, crystal structure and antitubercular activity of benzothiazolylpyrimidine-5-carboxamides, European journal of medicinal chemistry 96 (2015) 30-46.

11. R.V. Chikhale, A.M. Pant, S.S. Menghani, P.B. Khedekar, Development of dual inhibitors targeting DprE1 and AHAS for treatment of Mycobacterium tuberculosis infection, BMC Infectious Diseases 14(3) (2014) E24.

12. R.V. Chikhale, A.M. Pant, S.S. Menghani, P.G. Wadibhasme, P.B. Khedekar, Facile and efficient synthesis of benzoxazole derivatives using novel catalytic activity of PEG-SO $3 \mathrm{H}$, Arabian Journal of Chemistry.

13. D.R. Huron, M.E. Gorre, A.J. Kraker, C.L. Sawyers, N. Rosen, M.M. Moasser, A novel pyridopyrimidine inhibitor of abl kinase is a picomolar inhibitor of Bcrabl-driven K562 cells and is effective against STI571resistant Bcr-abl mutants, Clinical cancer research 9(4) (2003) 1267-1273.

14. K. Nakayama, H. Kawato, J. Watanabe, M. Ohtsuka, K.-i. Yoshida, Y. Yokomizo, A. Sakamoto, N. Kuru, T. Ohta, K. Hoshino, MexAB-OprM specific efflux pump inhibitors in Pseudomonas aeruginosa. Part 3: Optimization of potency in the pyridopyrimidine series through the application of a pharmacophore model, Bioorganic \& medicinal chemistry letters 14(2) (2004) 475-479.

15. G.Z. Zheng, Y. Mao, C.-H. Lee, J.K. Pratt, J.R. Koenig, R.J. Perner, M.D. Cowart, G.A. Gfesser, S. McGaraughty, K.L. Chu, Adenosine kinase inhibitors: 
polar 7-substitutent of pyridopyrimidine derivatives improving their locomotor selectivity, Bioorganic \& medicinal chemistry letters 13(18) (2003) 3041-3044.

16. T. Saurat, F. Buron, N. Rodrigues, M.L. de Tauzia, L. Colliandre, S. Bourg, P. Bonnet, G. Guillaumet, M. Akssira, A. Corlu, C. Guillouzo, P. Berthier, P. Rio, M.L. Jourdan, H. Benedetti, S. Routier, Design, Synthesis, and Biological Activity of Pyridopyrimidine Scaffolds as Novel PI3K/mTOR Dual Inhibitors, Journal of Medicinal Chemistry 57(3) (2014) 613-631.

17. S. Labadie, K. Barrett, W.S. Blair, C. Chang, G. Deshmukh, C. Eigenbrot, P. Gibbons, A. Johnson, J.R. Kenny, P.B. Kohli, M. Liimatta, P.J. Lupardus, S. Shia, M. Steffek, S. Ubhayakar, A. van Abbema, M. Zak, Design and evaluation of novel 8-oxopyridopyrimidine Jak1/2 inhibitors, Bioorg Med Chem Lett 23(21) (2013) 5923-30.

18. O.A. Mizenina, M.M. Moasser, S-phase inhibition of cell cycle progression by a novel class of pyridopyrimidine tyrosine kinase inhibitors, Cell Cycle 3(6) (2004) 794-801.

19. S.M. Rajesh, R.S. Kumar, L.A. Libertsen, S. Perumal, P. Yogeeswari, D. Sriram, A green expedient synthesis of pyridopyrimidine-2-thiones and their antitubercular activity, Bioorganic \& Medicinal Chemistry Letters 21(10) (2011) 3012-3016.

20. R.V. Chikhale, M.A. Barmade, P.R. Murumkar, M.R. Yadav, Overview of the development of DprE1 inhibitors for combating the menace of tuberculosis, Journal of medicinal chemistry 61(19) (2018) 85638593.

21. A.R. Saundane, K. Vijaykumar, A.V. Vaijinath, P. Walmik, Synthesis, antimicrobial and antioxidant activities of some new indole derivatives containing pyridopyrimidine and pyrazolopyridine moieties, Medicinal Chemistry Research 22(2) (2013) 806-817.

22. C.G. Dave, M.C. Shukla, Pyridopyrimidines: Part IXSynthesis and antibacterial activity of 2-methylthio-6phenylazo-5, 7-dimethylpyrido [2, 3-d] pyrimidin-4 (3H)-ones, (2000).

23. W. Ribble, W.E. Hill, U.A. Ochsner, T.C. Jarvis, J.W. Guiles, N. Janjic, J.M. Bullard, Discovery and analysis of $4 \mathrm{H}$-pyridopyrimidines, a class of selective bacterial protein synthesis inhibitors, Antimicrob Agents Chemother 54(11) (2010) 4648-57.

24. J.W. Guiles, A. Toro, U.A. Ochsner, J.M. Bullard, Development of 4H-pyridopyrimidines: a class of selective bacterial protein synthesis inhibitors, Org Med Chem Lett 2(1) (2012) 5.
25. M.A.E.A. Elâ€ Remaily, A. Ali, O. El Hady, H. Salah Abo Zaid, E. Abd Elâ€ Raheem, Synthesis and in vitro Antibacterial Activity of Some Novel Fused Pyridopyrimidine Derivatives, Journal of Heterocyclic Chemistry.

26. M.A. Silvers, G.T. Robertson, C.M. Taylor, G.L. Waldrop, Design, synthesis, and antibacterial properties of dual-ligand inhibitors of acetyl-CoA carboxylase, J Med Chem 57(21) (2014) 8947-59.

27. M. Brylinski, G.L. Waldrop, Computational Redesign of Bacterial Biotin Carboxylase Inhibitors Using Structure-Based Virtual Screening of Combinatorial Libraries, Molecules 19(4) (2014) 4021-4045.

28. C.Z. Blanchard, Y.M. Lee, P.A. Frantom, G.L. Waldrop, Mutations at four active site residues of biotin carboxylase abolish substrate-induced synergism by biotin, Biochemistry 38(11) (1999) 3393-3400.

29. C.Z. Blanchard, D. Amspacher, R. Strongin, G.L. Waldrop, Inhibition of biotin carboxylase by a reaction intermediate analog: implications for the kinetic mechanism, Biochemical and biophysical research communications 266(2) (1999) 466-471.

30. M.M. Bradford, A rapid and sensitive method for the quantitation of microgram quantities of protein utilizing the principle of protein-dye binding, Analytical biochemistry 72(1-2) (1976) 248-254.

31. [31] W.W. Cleland, [6] Statistical analysis of enzyme kinetic data, Methods in enzymology 63 (1979) 103 138.

32. M. Wikler, Clinical and Laboratory Standards Institute. Methods for dilution antimicrobial susceptibility tests for bacteria that grow aerobically; approved standard, (2009).

33. K. Drlica, The mutant selection window and antimicrobial resistance, Journal of Antimicrobial Chemotherapy 52(1) (2003) 11-17.

34. R. Chikhale, S. Thorat, A. Pant, A. Jadhav, K.C. Thatipamula, R. Bansode, G. Bhargavi, N. Karodia, M. Rajasekharan, A. Paradkar, Design, synthesis and pharmacological evaluation of pyrimidobenzothiazole3-carboxylate derivatives as selective L-type calcium channel blockers, Bioorganic \& medicinal chemistry 23(20) (2015) 6689-6713.

35. S. Chakravarty, S. Dugar, J.J. Perumattam, G.F. Schreiner, D.Y. Liu, J.A. Lewicki, Quinazoline derivatives as medicaments, Google Patents, 2002.

36. J.J. Li, Fischer-Speier esterification, Name Reactions, Springer2003, pp. 139-139. 